\title{
Effect of magnetic field on the time-dependent flow due to a disk with non-torsional oscillation and a Newtonian fluid at infinity rotating about distinct axes
}

\author{
H VOLKAN ERSOY \\ Department of Mechanical Engineering, Yildiz Technical University, 34349 Istanbul, Turkey \\ e-mail: ersoyhv@yahoo.com
}

MS received 30 September 2018; revised 7 April 2019; accepted 12 April 2019; published online 27 May 2019

\begin{abstract}
This work aims to study the effect of magnetic field on the time-dependent flow of an insulated disk executing non-torsional oscillation in its own plane and a Newtonian fluid at infinity while they are initially rotating at the same speed about two vertical axes. It is shown that the presence of a magnetic field causes a resistive force and the required time to reach the periodic state becomes shorter. Further, it is demonstrated that there is an excellent agreement between the exact solution that is appropriate for all values of time and the periodic solution that is valid after the periodicity of the flow starts.
\end{abstract}

Keywords. Magnetohydrodynamics; Newtonian fluid; oscillating disk; non-coaxial rotation; exact solution; periodic flow.

\section{Introduction}

After the pioneering research of Courier [1] that studied the steady flow caused by the rotation of a disk and a Newtonian fluid at infinity about two parallel vertical axes, the extensions under the influence of different physical conditions of this type of flow to the case of a disk with nontorsional oscillation in its own plane were carried out by several authors [2-12]. Kasiviswanathan and Rao [2], Hayat et al [3] and Guria et al [4] studied the periodic flow occurred by the oscillation of a porous disk while the porous disk and a Newtonian fluid at infinity are rotating at the same speed. Erdoğan [5] examined the time-dependent flow generated by the non-concentric rotation of a disk with non-torsional oscillations in its own plane and a fluid at infinity while they are rotating coaxially. Hayat et al [6] extended the analysis in [5] to the case of a porous disk for a second grade fluid with the small material modulus. The unsteady flows developed by the application of a magnetic field were investigated in [7-11]. Hayat et al [7] were the first to study the effect of magnetic field and presented an exact solution for a porous disk. Hayat et al [8] investigated the unsteady flow for the generalized non-torsional oscillations. The effect of Hall current on the flow of a porous disk was carried out by Hayat et al [9]. Hayat et al [10] carried out an analysis for the problem in [6] when a magnetic field is applied. Wang and $\mathrm{Wu}$ [11] obtained a numerical solution for the flow of a third-order fluid with slip condition in the case of a disk subjected to uniform suction or blowing. Apart from the above papers, Ersoy
[12] considered a different initial flow and studied the unsteady flow occurred after the disk performs non-torsional oscillation in its own plane while the disk and the fluid at infinity are initially rotating at the same speed about non-concentric axes.

The goal of this paper is to extend the analysis in [12] to the magnetohydrodynamic flow. Such a flow model has an application in the areas of earthquake and ocean engineering. Since the aim is to investigate the effect of magnetic field, it is focused on how both the velocity field and the shear stresses at the disk change with the magnetic parameter $N$. The exact solution corresponding to the flow is compared to a periodic solution of the flow field and an excellent agreement of the results obtained from both the methods is observed.

\section{Problem description and basic equations}

In a Cartesian coordinate system, let us consider an electrically conducting and incompressible Newtonian fluid filling the semi-infinite space. A non-conductive infinite disk is located at $z=0$. A uniform magnetic induction $\mathbf{B}_{0}$ is applied perpendicular to the disk. The induced magnetic field is neglected under the assumption of a small magnetic Reynolds number. While the disk and the fluid at infinity are initially rotating at the same speed $\Omega$ about the $z$ - and $z^{\prime}$ - axes, respectively, the disk also begins to execute non-torsional oscillation in its own plane with the velocity $\mathbf{U}=\left(U_{x} \sin n t, U_{y} \sin n t\right)$, where $n$ denotes the frequency of 
the oscillation and the distance between the axes is symbolized by $\ell$. The flow configuration is shown in figure 1 .

The continuity and momentum equations are

$$
\begin{gathered}
\nabla \cdot \mathbf{v}=0, \\
\rho \frac{D \mathbf{v}}{D t}=\nabla \cdot \mathbf{T}+\mathbf{J} \times \mathbf{B},
\end{gathered}
$$

where $\mathbf{v}$ is the velocity vector, $\rho$ the density, $D / D t$ the material time derivative, $\mathbf{T}$ the Cauchy stress tensor, $\mathbf{J}=$ $\left(J_{x}, J_{y}, J_{z}\right)$ the current density, $\mathbf{B}$ the magnetic induction.

The initial and boundary conditions are

$$
\begin{aligned}
& u=-\Omega y+\hat{f}(z), \quad v=\Omega x+\hat{g}(z), \quad \text { at } \quad t=0 \quad \text { for } \\
& \quad z \geq 0,
\end{aligned}
$$

$$
\begin{aligned}
& u=-\Omega y+U_{x} \sin n t, \quad v=\Omega x+U_{y} \sin n t, \quad \text { at } \quad z=0 \\
& \text { for } \quad t \geq 0, \\
& u=-\Omega(y-\ell), \quad v=\Omega x, \quad \text { at } \quad z \rightarrow \infty \text { for } t \geq 0,
\end{aligned}
$$

where $u$ and $v$ are the components in the $x$ - and $y$-directions of the velocity vector $\mathbf{v}$. The functions $\hat{f}(z)$ and $\hat{g}(z)$ symbolize the components of translational velocity corresponding to the steady magnetohydrodynamic flow produced by the rotation of a disk and a Newtonian fluid at infinity about the $z$ - and $z^{\prime}$-axes in figure 1 . The velocity field, compatible with the continuity equation (1), can be written as follows:

$$
u=-\Omega y+f(z, t), \quad v=\Omega x+g(z, t) .
$$

Here, $f(z, t)$ and $g(z, t)$ are the $x$-and $y$-components of the unsteady translational velocity, respectively.

By means of $\mathbf{J}=\sigma(\mathbf{E}+\mathbf{v} \times \mathbf{B})$, we obtain $J_{x}=\sigma\left(E_{x}+v B_{0}\right), J_{y}=\sigma\left(E_{y}-u B_{0}\right), J_{z}=\sigma E_{z}$, where $\sigma$ is the electrical conductivity of the fluid and $\mathbf{E}=\left(E_{x}, E_{y}, E_{z}\right)$ is the electric field. Since the disk is insulated, we get $J_{z}=$ 0 and $E_{z}=0$. Using $\nabla \times \mathbf{E}=-\frac{\partial \mathbf{B}}{\partial t}$, we find that $E_{x}$ and $E_{y}$ are independent of $z$. Taking into account the fact that the fluid at infinity has no shear stress, substituting Eq. (6) into Eq. (2) yields

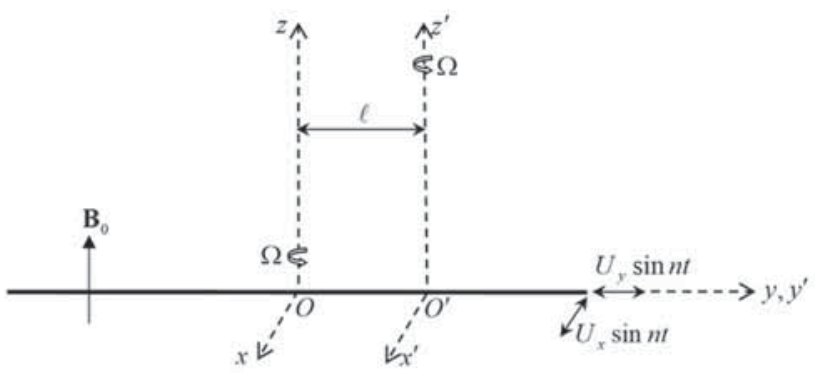

Figure 1. Flow geometry.

$$
\begin{aligned}
& \mu \frac{\partial^{2} f}{\partial z^{2}}-\rho \frac{\partial f}{\partial t}+\rho \Omega g-\sigma B_{0}^{2} f=-\sigma B_{0}^{2} \Omega \ell \\
& \mu \frac{\partial^{2} g}{\partial z^{2}}-\rho \frac{\partial g}{\partial t}-\rho \Omega f-\sigma B_{0}^{2} g=-\rho \Omega^{2} \ell
\end{aligned}
$$

where $\mu$ is the dynamic viscosity of the fluid, $\rho$ the density and $B_{0}$ the magnitude of the applied magnetic induction. The functions $\hat{f}(z)$ and $\hat{g}(z)$ are easily found by Eq. (2) in the complex form as follows:

$$
\hat{f}(z)+i \hat{g}(z)=\Omega \ell[1-\exp (-\sqrt{P} z)],
$$

where $P=\frac{\sigma B_{0}^{2}+\rho \Omega i}{\mu}$.

The dimensionless variables are presented as follows:

$$
\begin{aligned}
& \bar{f}(\zeta, \tau)=\frac{f(z, t)}{\Omega \ell}, \quad \bar{g}(\zeta, \tau)=\frac{g(z, t)}{\Omega \ell}, \\
& \tilde{F}(\zeta, \tau)=\bar{f}(\zeta, \tau)+i \bar{g}(\zeta, \tau)-1, \\
& \zeta=\sqrt{\frac{\rho \Omega}{2 \mu}} z, \quad \tau=\Omega t, \quad k=\frac{n}{\Omega}, \quad N=\frac{\sigma B_{0}^{2}}{\rho \Omega}, \\
& V_{x}=\frac{U_{x}}{\Omega \ell}, \quad V_{y}=\frac{U_{y}}{\Omega \ell},
\end{aligned}
$$

where $\zeta$ is the dimensionless distance, $\tau$ the dimensionless time, $k$ the ratio of the frequency of oscillation to the angular velocity of the disk, $N$ the magnetic parameter, $V_{x}$ and $V_{y}$ the dimensionless velocity amplitudes in the $x$ and $y$ directions. In terms of these dimensionless quantities, we obtain the non-dimensional governing equation and the corresponding initial and boundary conditions as follows:

$$
\begin{gathered}
\frac{\partial^{2} \tilde{F}}{\partial \zeta^{2}}-2 \frac{\partial \tilde{F}}{\partial \tau}-2(N+i) \tilde{F}=0, \\
\tilde{F}(\zeta, 0)=-\exp [-\sqrt{2(N+i)} \zeta] \text { for } \zeta \geq 0, \\
\tilde{F}(0, \tau)=\left(V_{x}+i V_{y}\right) \sin k \tau-1 \text { for } \tau \geq 0, \\
\tilde{F}(\infty, \tau)=0 \text { for } \tau \geq 0 .
\end{gathered}
$$

\subsection{Exact solution}

Putting

$$
\tilde{F}(\zeta, \tau)=H(\zeta, \tau) \exp [-(N+i) \tau]
$$

we get

$$
\begin{gathered}
\frac{\partial^{2} H}{\partial \zeta^{2}}-2 \frac{\partial H}{\partial \tau}=0 \\
H(\zeta, 0)=-\exp [-\sqrt{2(N+i)} \zeta] \text { for } \zeta \geq 0, \\
H(0, \tau)=\left[\left(V_{x}+i V_{y}\right) \sin k \tau-1\right] \exp [(N+i) \tau] \text { for } \\
\tau \geq 0,
\end{gathered}
$$




$$
H(\infty, \tau)=0 \quad \text { for } \quad \tau \geq 0 .
$$

The Laplace transform of $H(\zeta, \tau)$ is defined by

$$
\bar{H}(\zeta, s)=\int_{0}^{\infty} H(\zeta, \tau) \exp (-s \tau) d \tau .
$$

Taking the Laplace transforms of Eq. (15) and Eqs. (17)(18), one obtains

$$
\begin{gathered}
\bar{H}^{\prime \prime}-2 s \bar{H}=2 \exp [-\sqrt{2(N+i)} \zeta] \\
\bar{H}(0, s)=\frac{\left(V_{x}+i V_{y}\right) k}{(s-N-i)^{2}+k^{2}}-\frac{1}{s-N-i}, \\
\bar{H}(\infty, s)=0 .
\end{gathered}
$$

The solution of Eq. (20) subject to Eqs. (21)-(22) is

$$
\begin{aligned}
\bar{H}(\zeta, s)= & \frac{\left(V_{x}+i V_{y}\right) k}{(s-N-i)^{2}+k^{2}} \exp (-\sqrt{2 s \zeta}) \\
& -\frac{1}{s-N-i} \exp [-\sqrt{2(N+i)} \zeta] .
\end{aligned}
$$

The Laplace inversion of Eq. (23) gives

$$
\begin{aligned}
& \bar{f}(\zeta, \tau)+i \bar{g}(\zeta, \tau)=1-\exp [-\sqrt{2(N+i)} \zeta] \\
& -\frac{1}{4}\left(V_{y}-i V_{x}\right)[\exp (-i k \tau) A(\zeta, \tau)-\exp (i k \tau) B(\zeta, \tau)],
\end{aligned}
$$

where

$$
\begin{aligned}
A(\zeta, \tau) & =A_{1}(\zeta, \tau)+A_{2}(\zeta, \tau), \\
A_{1}(\zeta, \tau) & =\exp \left[I_{1}(\zeta)\right] \operatorname{erfc}\left[J_{1}(\zeta, \tau)\right], \\
I_{1}(\zeta) & =\sqrt{2(N+i(1-k)) \zeta,} \\
J_{1}(\zeta, \tau) & =\frac{\zeta}{\sqrt{2 \tau}}+\sqrt{(N+i(1-k)) \tau}, \\
A_{2}(\zeta, \tau) & =\exp \left[I_{2}(\zeta)\right] \operatorname{erfc}\left[J_{2}(\zeta, \tau)\right], \\
I_{2}(\zeta) & =-\sqrt{2(N+i(1-k))}, \\
J_{2}(\zeta, \tau) & =\frac{\zeta}{\sqrt{2 \tau}}-\sqrt{(N+i(1-k)) \tau}, \\
B(\zeta, \tau) & =B_{1}(\zeta, \tau)+B_{2}(\zeta, \tau), \\
B_{1}(\zeta, \tau) & =\exp \left[I_{3}(\zeta)\right] \operatorname{erfc}\left[J_{3}(\zeta, \tau)\right], \\
I_{3}(\zeta) & =\sqrt{2(N+i(1+k)) \zeta,} \\
J_{3}(\zeta, \tau) & =\frac{\zeta}{\sqrt{2 \tau}}+\sqrt{(N+i(1+k)) \tau}, \\
B_{2}(\zeta, \tau) & =\exp \left[I_{4}(\zeta)\right] \operatorname{erfc}\left[J_{4}(\zeta, \tau)\right], \\
I_{4}(\zeta) & =-\sqrt{2(N+i(1+k))} \zeta, \\
J_{4}(\zeta, \tau) & =\frac{\zeta}{\sqrt{2 \tau}}-\sqrt{(N+i(1+k)) \tau} .
\end{aligned}
$$

\subsection{Periodic solution}

In order to present a solution corresponding to the periodic flow, we consider the following form:

$$
\tilde{F}(\zeta, \tau)=\tilde{F}_{0}(\zeta)+\tilde{F}_{1}(\zeta) \cos k \tau+\tilde{F}_{2}(\zeta) \sin k \tau,
$$

where $\tilde{F}_{0}(z)$ corresponds to the case of $n=0$ and is the same as the dimensionless case of Eq. (4). Substituting Eq. (28) into Eq. (10), we obtain 
(a)

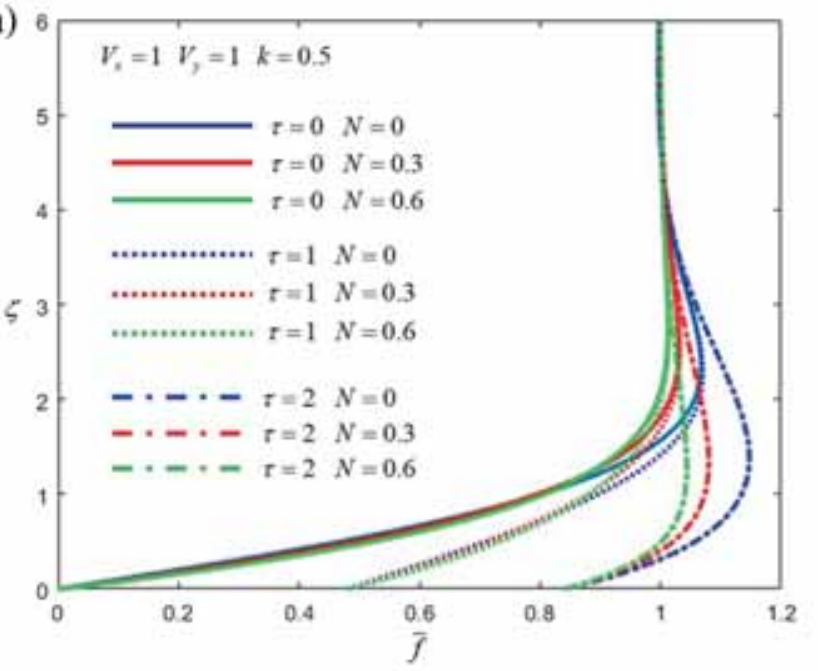

(c)

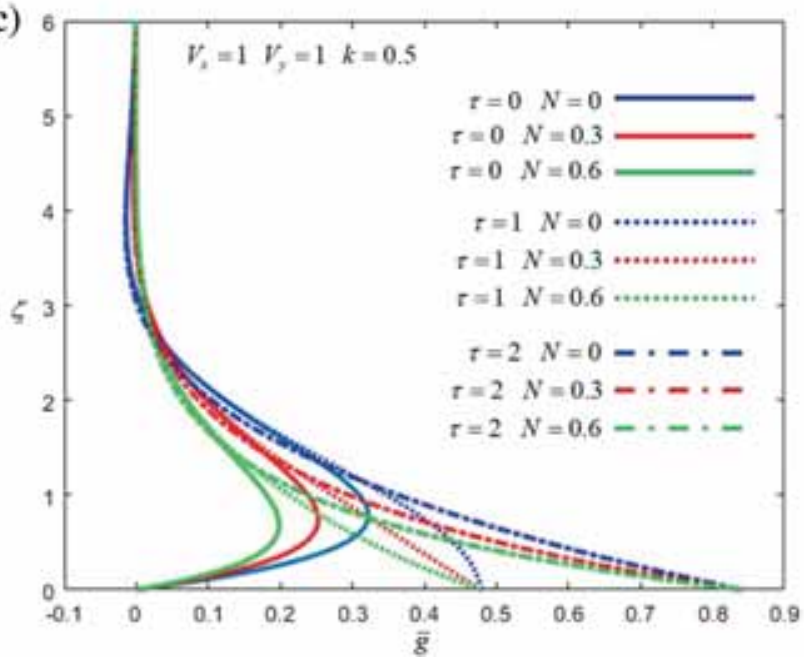

(b)

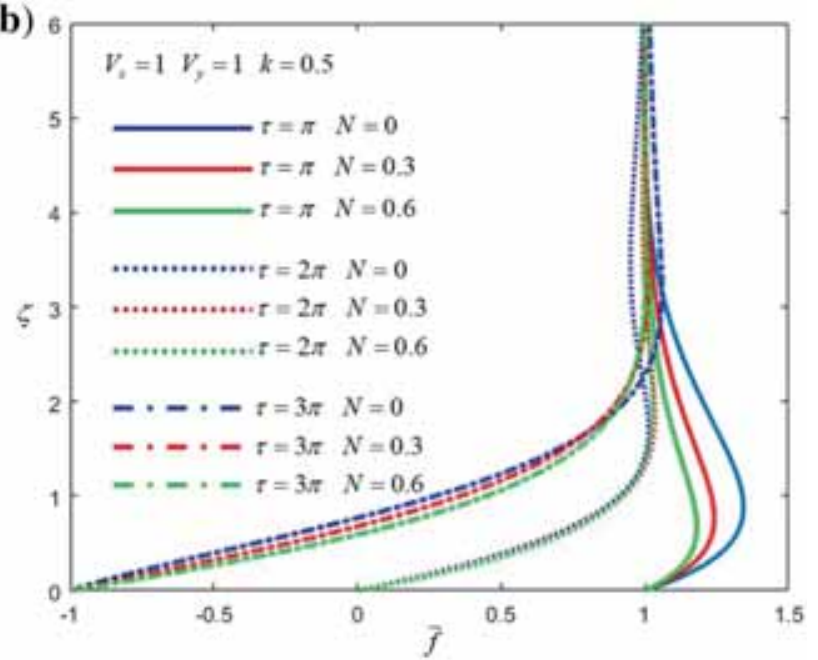

(d)

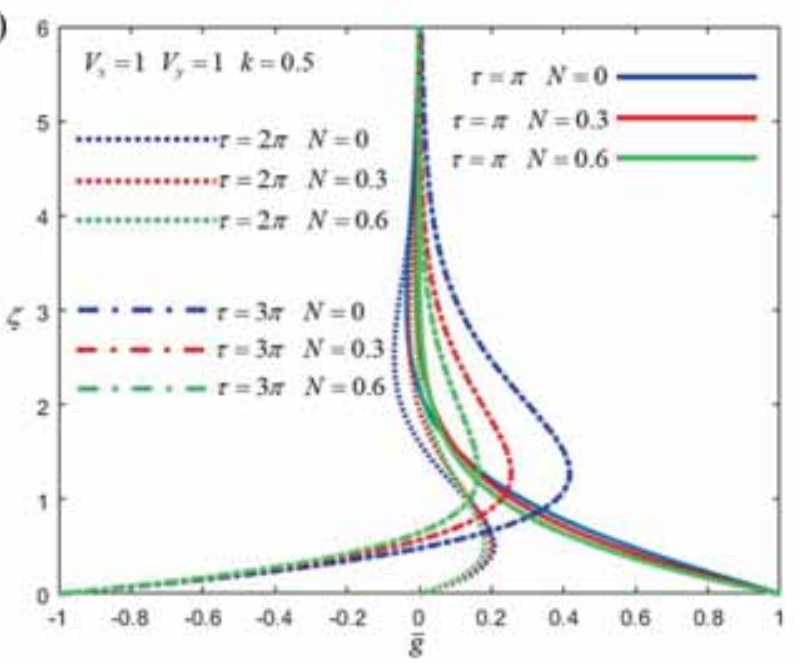

Figure 2. Variation of $\bar{f}$ and $\bar{g}$ with $\zeta$ for $V_{x}=1, V_{y}=1, k=0.5(N=0,0.3,0.6)$.

$$
\begin{gathered}
\tilde{F}_{0}^{\prime \prime}-2(N+i) \tilde{F}_{0}=0, \\
\tilde{F}_{1}^{\prime \prime}-2(N+i) \tilde{F}_{1}-2 k \tilde{F}_{2}=0, \\
\tilde{F}_{2}^{\prime \prime}-2(N+i) \tilde{F}_{2}+2 k \tilde{F}_{1}=0,
\end{gathered}
$$

with

$$
\begin{array}{lll}
\tilde{F}_{0}(0)=-1, & \tilde{F}_{1}(0)=0, & \tilde{F}_{2}(0)=V_{x}+i V_{y} \\
\tilde{F}_{0}(\infty)=0, & \tilde{F}_{1}(\infty)=0, & \tilde{F}_{2}(\infty)=0
\end{array}
$$

It should be pointed out that the initial condition of the problem presented in Eq. (4) is equal to the dimensional form of the solution of Eq. (29). After the solutions of Eqs. (29)-(31) satisfying Eq. (32), we obtain

$$
\begin{aligned}
& \bar{f}(\zeta, \tau)+i \bar{g}(\zeta, \tau)=1-\exp [-\sqrt{2(N+i)} \zeta] \\
& -\frac{1}{2}\left(V_{y}-i V_{x}\right)\{\exp [M(\zeta, \tau)]-\exp [Q(\zeta, \tau)]\},
\end{aligned}
$$

and

$$
\begin{aligned}
& \bar{T}_{x z}(\zeta, \tau)+i \bar{T}_{y z}(\zeta, \tau)=\sqrt{2(N+i)} \exp [-\sqrt{2(N+i)} \zeta] \\
& +\frac{\sqrt{2}}{2}\left(V_{y}-i V_{x}\right)\{\sqrt{N+i(1-k)} \exp [M(\zeta, \tau)] \\
& \quad-\sqrt{N+i(1+k)} \exp [Q(\zeta, \tau)]\},
\end{aligned}
$$

where

$$
\begin{aligned}
M(\zeta, \tau) & =-\sqrt{2(N+i(1-k))} \zeta-i k \tau, \\
Q(\zeta, \tau) & =-\sqrt{2(N+i(1+k))} \zeta+i k \tau .
\end{aligned}
$$

It should be emphasized that the periodic solution is acceptable after the periodicity of the flow starts. Figure 4 shows a comparison between the exact and periodic 

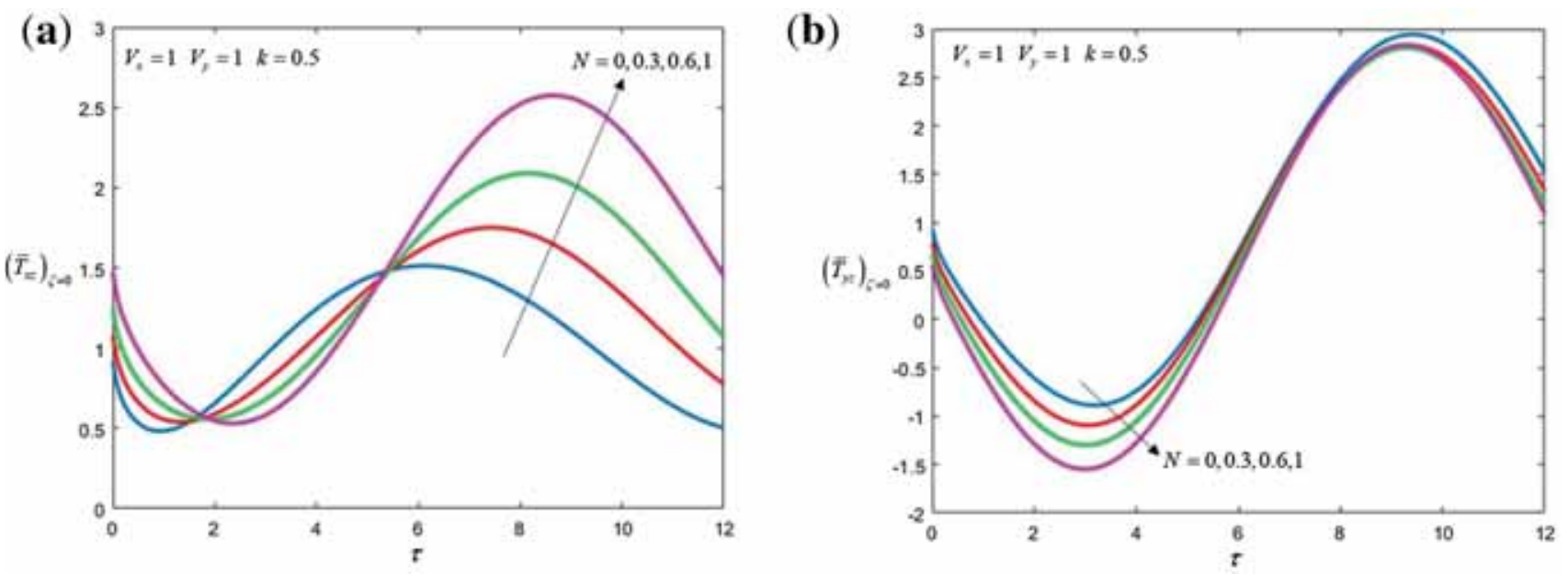

Figure 3. Variation of $\left(\bar{T}_{x z}\right)_{\zeta=0}$ and $\left(\bar{T}_{y z}\right)_{\zeta=0}$ with $\tau$ for $V_{x}=1, V_{y}=1, k=0.5(N=0,0.3,0.6,1)$.

(a)

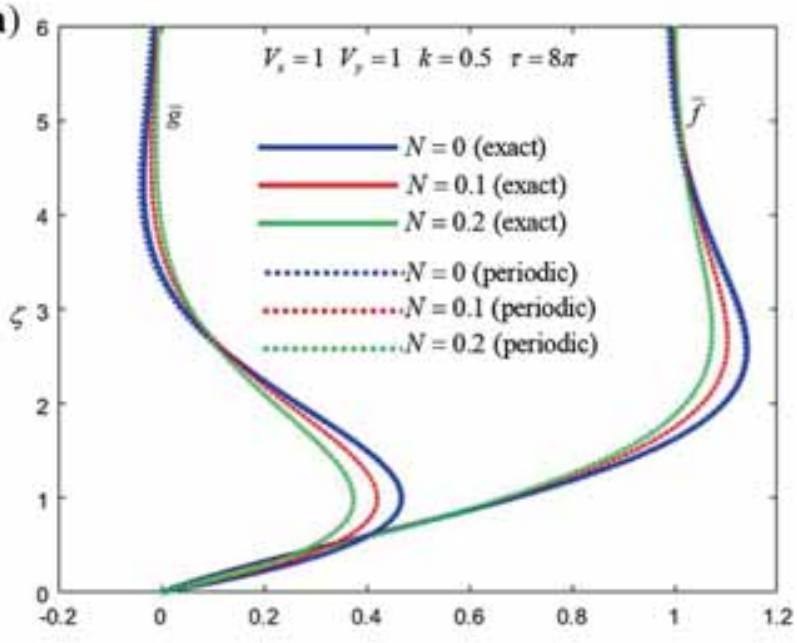

(b)

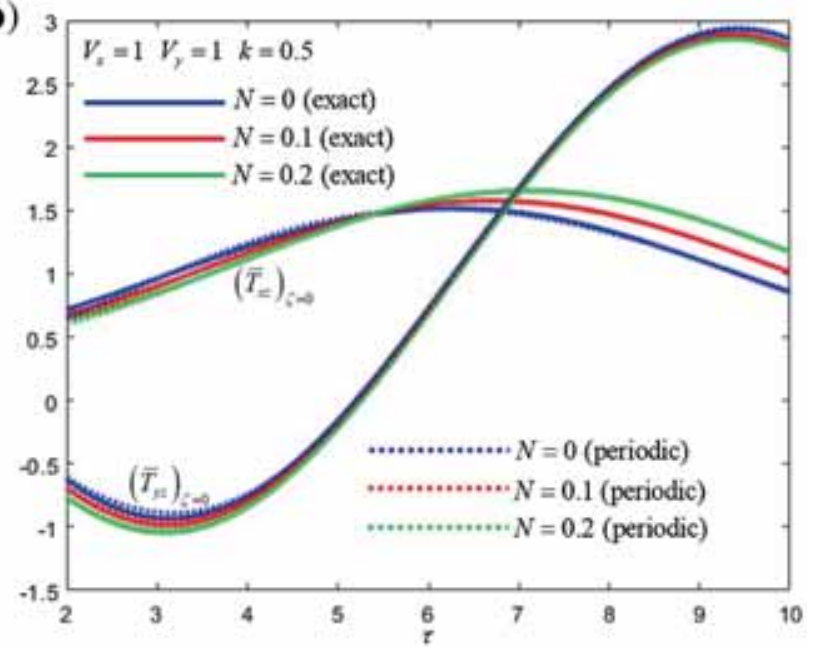

Figure 4. Comparison of exact and periodic solutions for $V_{x}=1, V_{y}=1, k=0.5(N=0,0.1,0.2)$.

solutions. Thus, it is shown that the flow reaches its periodic state after the initial transients decay.

\section{Results and discussion}

In this paper, while the disk and the fluid at infinity are initially rotating about non-concentric axes under the application of a magnetic field, the unsteady flow developed after the disk begins to execute non-torsional oscillation in its own plane is studied. The exact solution derived using the Laplace transform technique includes the velocity field and shear stresses at the disk.

Figure 2 indicates that an increase in the magnetic parameter $N$ causes a decrease in the $x$ - and $y$-components of the translational velocity. At a certain distance from the disk, the axes of rotation of the fluid layers get closer to those of the fluid at infinity. This effect is a conclusion of the Lorentz force which has a tendency to slow down the motion of the fluid. Figure 3 displays that the shear stresses at the disk have larger changes when the magnetic parameter $N$ increases. Figure 4 illustrates a comparison between the exact solution that corresponds to all times and the periodic solution that is valid after the periodic flow starts in the fluid. Bearing in mind that the periodic solution is not valid for small times, an excellent agreement between the two solutions for large times is found. It should be noted that the flow is considered to be periodic at $\tau=8 \pi$ for $V_{x}=1, V_{y}=1, k=0.5$ as observed from figure 4(a) and the dimensionless time in figure 4(b) is started from a small value $(\tau=2)$ to discern the difference between the two solutions since the difference between the exact and periodic curves vanishes as the time elapses. 


\section{Conclusions}

In this study, the emphasis is given to the effect of applied magnetic field. The results found in the absence of magnetic field [12] can be recovered by setting $N=0$. As expected, the application of the magnetic field results in the appearance of a Lorentz force which resists the flow. Hence a reduction in the velocity is observed and the amplitudes of the shear stresses at the disk become larger. It is proved that the required time for the periodicity of the flow becomes shorter with the presence of a magnetic field. It is observed that the applied magnetic field causes the boundary layer to thin.

\section{Acknowledgements}

The author would like to express his sincere thanks to the referees for their valuable comments and suggestions.

\section{References}

[1] Coirier J 1972 Rotations non coaxiales d'un disque et d'un fluide à l'infini. J. Méc. 11(2): 317-340

[2] Kasiviswanathan S R and Rao A R 1987 An unsteady flow due to eccentrically rotating porous disk and a fluid at infinity. Int. J. Eng. Sci. 25(11-12): 1419-1425

[3] Hayat T, Asghar S and Siddiqui A M 1999 Unsteady flow of an oscillating porous disk and a fluid at infinity. Meccanica 34(4): 259-265
[4] Guria M, Das B K and Jana R N 2007 Oscillatory flow due to eccentrically rotating porous disk and a fluid at infinity. Meccanica 42(5): 487-493

[5] Erdoğan M E 2000 Flow induced by non-coaxial rotation of a disk executing non-torsional oscillations and a fluid rotating at infinity. Int. J. Eng. Sci. 38(2): 175-196

[6] Hayat T, Ellahi R and Asghar S 2004 Flow induced by noncoaxial rotation of a porous disk executing non-torsional oscillations and a second grade fluid rotating at infinity. Appl. Math. Model. 28(6): 591-605

[7] Hayat T, Zamurad M, Asghar S and Siddiqui A M 2003 Magnetohydrodynamic flow due to non-coaxial rotations of a porous oscillating disk and a fluid at infinity. Int. J. Eng. Sci. 41(11): 1177-1196

[8] Hayat T, Ellahi R and Asghar S 2004 Unsteady periodic flows of a magnetohydrodynamic fluid due to noncoaxial rotations of a porous disk and a fluid at infinity. Math. Comput. Model. 40(1-2): 173-179

[9] Hayat T, Ellahi R and Asghar S 2008 Hall effects on unsteady flow due to non-coaxially rotating disk and a fluid at infinity. Chem. Eng. Commun. 195(8): 958-976

[10] Hayat T, Ellahi R and Asghar S 2007 Unsteady magnetohydrodynamic non-Newtonian flow due to non-coaxial rotations of disk and a fluid at infinity. Chem. Eng. Commun. 194(1): 37-49

[11] Wang Y and Wu W 2007 Time-dependent magnetohydrodynamic flow induced by non-coaxial rotations of a nontorsionally oscillating porous plate and a third-order fluid at infinity. Math. Comput. Model. 46(9-10): 1277-1293

[12] Ersoy H V 2017 Unsteady flow due to a disk executing nontorsional oscillation and a Newtonian fluid at infinity rotating about non-coaxial axes. Sadhana - Acad. Proc. Eng. Sci. 42(3): 307-315 